\title{
WBSGEN - Wissensbasiertes System für das Genehmigungsverfahren baulicher Anlagen
}

Helmut Waldorf, Essen

Die Planung und Durchführung von Bauvorhaben zu Gebäuden und Anlagen erfordert die Beachtung zahlreicher Gesetze, Verordnungen und Normen. Durch die Fülle der infrage kommenden Vorschriften und durch die Unübersichtlichkeit der darin formulierten Anforderungen wird die Erstellung von Genehmigungsanträgen für Bauvorhaben zu einem aufwendigen Problem.

WBSGEN unterstützt in der Planungsphase und bei der Formulierung des Genehmigungsantrags:

Zunächst wird das Bauvorhaben grob beschrieben. Dazu werden Angaben zum vorgesehenen Verfahren (z.B. Lagern), zum eingesetzten Stoff (z.B. Schwefelsäure), zu Volumina und Art der Lagerung gemacht. Anschließend ermittelt das System die für das vorliegende Bauvorhaben relevanten Gesetze - im derzeitigen Prototyp noch beschränkt auf das Wasserhaushaltsgesetz - und die darin enthaltenen Detailanforderungen für die bauliche Ausführung und den späteren Betrieb, gyf. mit möglichen Alternativen.

Erreicht wurde dieses über einen wissensbasierten Ansatz: Ein Gesetz wird in Einzelanforderungen zerlegt, die in einer objekthierarchie angeordnet werden. Die Relevanzkriterien für jedes objekt werden durch entsprechende Regeln beschrieben.

Die Implementierung erfolgte mit Hilfe der IBM Prolog Workbench, wesentliche Auswahlkriterien waren hierbei die gute Kooperation regelbasierter und objektorientierter Programmierung, der einfache Aufbau einer Benutzerschnittstelle unter dem Presentation Manager und die verfügbare Schnittstelle zum Database Manager. 